inflammatory cells $(\sim 1.8$-fold, $p<0.05)$ and $30 \%(p<0.05)$ more VCAM-1 positive blood vessels in AngII treated TG hearts. Furthermore, isolated TG endothelial cells recruited $\sim 2$-fold $(p<0.05)$ more leukocytes than WT upon AngII treatment.

Conclusion These results indicate there is a cell-specific role of endothelial Nox2 in the development of fibrosis. Endothelial Nox2 enhances AngII-induced cardiac fibrosis, possibly by enhancing inflammatory cell recruitment and influx via VCAM-1 expression. Although inflammatory cells may be important for the development of fibrosis, our results indicate that Nox2 in these cells is not essential for any pro-fibrotic effect.

\section{ENDOTHELIAL SPECIFIC INSULIN RESISTANCE PROMOTES THE DEVELOPMENT OF ATHEROSCLEROSIS}

doi:10.1136/heartjnl-2011-300198.139

${ }^{1} \mathrm{M} C$ Gage, ${ }^{1} \mathrm{~N}$ Yuldasheva, ${ }^{2} \mathrm{C}$ Jackson, ${ }^{1} \mathrm{M}$ Kearney, ${ }^{1} \mathrm{H}$ Imrie, ${ }^{1} \mathrm{H}$ Viswambharan, ${ }^{1} \mathrm{M}$ Kahn, ${ }^{1} \mathrm{~J}$ Smith, ${ }^{1} \mathrm{~S}$ Galloway, ${ }^{1} \mathrm{R}$ Cubbon, ${ }^{1} \mathrm{P}$ Sukumar, ${ }^{1} \mathrm{~A}$ Aziz, ${ }^{1} \mathrm{~S}$ Wheatcroft. ${ }^{1}$ Leeds University, Leeds, UK; ${ }^{2}$ University of Bristol, Bristol, UK

Background Global insulin resistance and endothelial dysfunction have been identified as predisposing factors for atherosclerosis. However, it is unclear whether selective insulin resistance in endothelial cells alone, is sufficient to promote atherosclerosis. Here we addressed this question by crossing Endothelial Specific Mutant Insulin Receptor Over-expressing (ESMIRO) mice with ApoE null mice. ESMIRO mice over-express a human insulin receptor with Ala-Thr1134 mutation in the tyrosine kinase domain (which disrupts insulin signalling) selectively in endothelial cells under the control of the tie-2 promoter/enhancer.

Methods Male ApoE ${ }^{-1}$ ESMIRO mice were compared with sexmatched littermate ApoE ${ }^{-1-}$ mice (both on a C57Bl6 background) after feeding a Western-style diet for 12 weeks.

Results ApoE ${ }^{-/}$ESMIRO mice were morphologically indistinguishable from $\mathrm{ApoE}^{-/-}$control littermates, with normal development and no difference between groups in body mass. Heart rate, systolic blood pressure, glucose tolerance, insulin sensitivity and fasting glucose levels were similar in ApoE-/-ESMIRO and $\mathrm{ApoE}^{-/-}$ mice. Aortic lipid deposition, assessed by en-face oil red $\bigcirc$ staining, was similar in $\mathrm{ApoE}^{-/-}$ESMIRO and $\mathrm{ApoE}^{-/-}$mice $(6.4 \% \pm 0.5 \%$ vs $5.8 \% \pm 0.5 \% ; \mathrm{p}=0.39)$. However, atherosclerotic lesion area in cross sections of aortic sinus was significantly increased in $\mathrm{ApoE}^{-1-}$ ESMIRO mice compared to $\mathrm{ApoE}^{-/-}$controls $(24.8 \% \pm 2.4 \%$ vs $16.6 \% \pm 2.4 \% ; \mathrm{p}=0.02)$. Absolute plaque size was also significantly increased in $\mathrm{ApoE}^{-1-}$ ESMIRO mice compared to ApoE controls (226448.9 $\pm 16154 \mu^{2}$ vs $149424.41 \pm 24221 \mu \mathrm{m}^{2}$; $\left.\mathrm{p}=0.01\right)$.

Conclusions Endothelial specific insulin resistance is sufficient to promote atherosclerosis and increase lesion area in ApoE null mice. This suggests that enhancing endothelial insulin sensitivity may be an appropriate target to prevent atherosclerosis in insulin-resistant conditions.

\section{IN VIVO DEPLETION OF ENDOGLIN RESULTS IN SIGNIFICANT LEFT VENTRICULAR REMODELLING}

doi:10.1136/heartjnl-2011-300198.140

${ }^{1} \mathrm{~B}$ J Davison, ${ }^{1} \mathrm{R}$ Redgrave, ${ }^{1} \mathrm{~B}$ Keavney, ${ }^{2} \mathrm{~A}$ Blamire, ${ }^{1} \mathrm{H}$ Arthur. ${ }^{1}$ Institute of Human Genetics, Newcastle University, Newcastle Upon Tyne, UK; ${ }^{2}$ Institute of Cellular Medicine, Newcastle University, Newcastle Upon Tyne, UK

Endoglin, a TGF $\beta$ co-receptor, is essential for cardiovascular development. However, endoglin also has an important role in fibrosis in adult life. Endoglin heterozygous mice have been shown to have reduced fibrosis in response to renal injury. They also have signifi- cantly reduced cardiac function following myocardial infarction. In rat cardiac fibroblasts, endoglin expression is up regulated following stimulation with angiotensin II and TGF $\beta$, resulting in reduced expression of MMP1 and increased expression of collagen I. These effects are inhibited by an endoglin specific antibody. Using our conditional endoglin knockout mice we sought to investigate the role of endoglin in cardiac healing following myocardial infarction. Adult Eng fl/fl Rosa26-Cre ${ }^{\text {ERT2 }}$ or control (Eng fl/fl) mice were treated with intraperitoneal injection of tamoxifen for 5 days to activate $\mathrm{Cre}{ }^{\text {ERT2 }}$ and deplete endoglin by Cre-lox recombination. Mice then underwent surgical coronary artery ligation or sham operation. Cine cardiac MRI was performed 28 days after injury. Measurement of left ventricular (LV) volumes and myocardial mass were made using ImageJ, and parameters of cardiac function were calculated. We found that LV volumes and mass were significantly increased $(p<0.001)$ and ejection fraction significantly reduced $(p=0.005)$ in endoglin deficient mice compared to controls. However, we also noticed LV volume and mass were increased in sham operated endoglin deficient mice. This led us to investigate the effect of endoglin knockdown on normal heart structure and function in adult mice. Cine cardiac MRI was therefore performed on mice without any surgical procedure after endoglin knockdown. We found that in the endoglin deficient mice, LV volume and mass were again significantly increased $(p<0.03)$. However, ejection fraction did not differ significantly from controls. These results demonstrate that depletion of endoglin results in significant left ventricular remodelling and suggest that endoglin plays an essential role in the maintenance of normal cardiac structure. The fact that cardiac function was preserved indicates that this is not a cardiomyopathic process and we hypothesise that the increased left ventricular volume in the endoglin-deficient mice may be the result of alterations in the extracellular matrix. We are currently investigating this potential molecular mechanism for left ventricular remodelling in the absence of endoglin.

\section{TISSUE FACTOR PATHWAY INHIBITOR REGULATES VASCULAR DEVELOPMENT IN ZEBRAFISH}

doi:10.1136/heartjnl-2011-300198.141

${ }^{1} \mathrm{E}$ W Holroyd, ${ }^{2} \mathrm{C}$ K Pierret, ${ }^{2} \mathrm{~V}$ M Bedell, ${ }^{3} \mathrm{~J}$ Townend, ${ }^{2} \mathrm{~S} \mathrm{C}$ Ekker, ${ }^{2} \mathrm{R} D$ Simari. ${ }^{1}$ Queen Elizabeth Hospital, Birmingham, UK; ${ }^{2}$ Mayo Clinic, Rochester, UK; ${ }^{3}$ Queen Elizabeth Hospital, Rochester, UK

Introduction Angiogenesis requires the coordinate regulation of multiple biological pathways, including haemostasis. Tissue factor pathway inhibitor (TFPI) is a potent anticoagulant molecule, inhibiting tissue factor-led coagulation. However, the role of TFPI in vascular development is unknown. Zebrafish (Danio rerio) provide a unique model system to study vascular development in vertebrates. Despite the divergence of jawed fish (teleosts) over 430 million years ago, there is notable conservation of the constituent molecules of the clotting cascade. Multiple features make this vertebrate model unique, including its genetic accessibility, the ability to titrate the degree of genetic knock-down, external embryonic development, and the transparent nature of the embryos.

Methods Using in-situ hybridisation techniques, we demonstrate TFPI expression during early vertebrate development. We then utilise transgenic fish with labelled endothelium (Fli1GFP) and erythrocytes (GATA1dsRed), to study in real time, concomitant fluorescent imaging of both structural development and dynamic blood flow observation, in living zebrafish embryos.

Results TFPI expression was identified at $24 \mathrm{~h}$ post fertilisation (hpf) in the pronephros (Abstract 141 figure 1-dark blue staining denotes TFPI expression; none seen in control embryos). Subsequently, TFPI mRNA became more abundant, localising to 


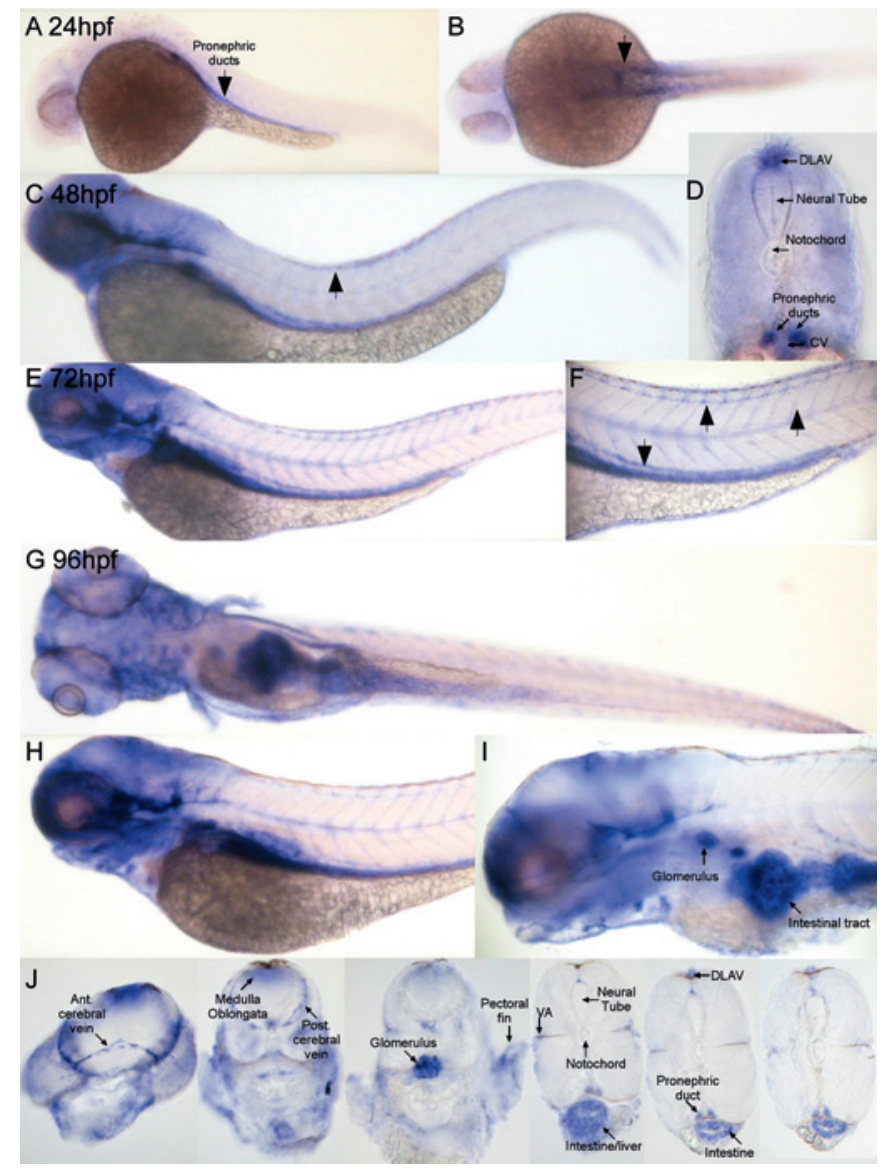

Abstract 141 Figure 1

developing kidney, gut, and vasculature. Morpholino (MO)-based knockdown of TFPI resulted in coagulopathy and disordered vascular development (Abstract 141 figure 2. left column: green endothelial Fli1GFP, right column: red erythrocytes. AB: uninjected controls. CD: MO controls. E-P: TFPI knockdown). Abnormally targetted (ie, vessels sprouting from normal site but growing in abnormal direction; grey arrows) and extra vessels (ie, superfluous vessels not seen in controls; white arrows in Abstract 141 figure

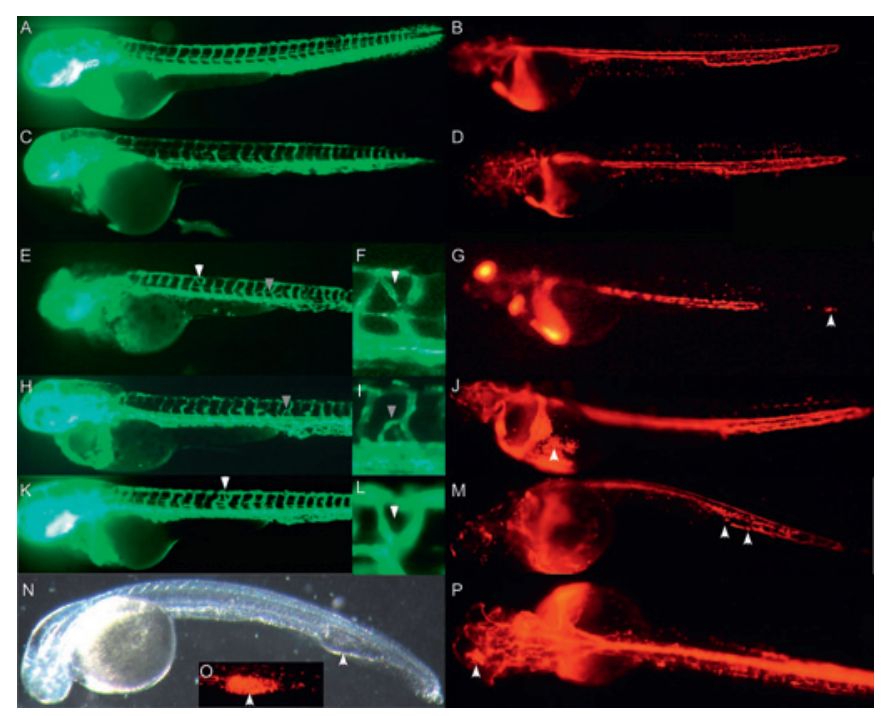

Abstract 141 Figure 2
2EFHIK\&L) were seen by $48 \mathrm{hpf}$. TFPI MO induced coagulopathy (a spontaneous clot or bleed; white arrows Abstract 141 figure 2GJMP) in $25.2 \pm 2.3 \% \quad(p<0.01$ cf. uninjected controls $)$ at $3 \mathrm{ng}$ and $23.8 \pm 5.8 \%(p<0.01)$ at $9 \mathrm{ng}$. Control embryos did not demonstrate significant signs of coagulopathy $(3.3 \pm 3.3 \%)$. Extra arteries occurred in $26.4 \pm 2.6 \% \quad(p<0.001$ by ANOVA cf. uninjected controls) of embryos injected with $3 \mathrm{ng}$ of TFPI MO. To further define the role of TFPI in vascular function, RNAi-mediated knockdown of TFPI was performed in human endothelial cells (EC). Knockdown of TFPI resulted in enhanced EC tube formation on Matrigel and EC migration in injury model associated with increased phosphorylation of Vascular Endothelial Growth Factor Receptor-2.

Conclusion These data represent the first demonstration of TFPI expression in zebrafish and the first description of a unique phenotype following TFPI knockdown. They support a model in which TFPI acts a molecular break to angiogenesis both in vivo, during early vertebrate embryogenesis, and in vitro in mature human endothelial cells, secondary to constitutive regulation of VEGF signalling.

\section{ATRIAL SOURCES OF REACTIVE OXYGEN SPECIES VARY WITH THE SUBSTRATE AND DURATION OF ATRIAL FIBRILLATION: IMPLICATIONS FOR THE ANTIARRHYTHMIC EFFECT OF STATINS}

doi:10.1136/heartjnl-2011-300198.142

${ }^{1} \mathrm{~S}$ Reilly, ${ }^{1} \mathrm{R}$ Jayaram, ${ }^{2} \mathrm{C}$ Anroniades, ${ }^{3} \mathrm{~S}$ Verheule, ${ }^{1} \mathrm{~K}$ M Channon, ${ }^{1} \mathrm{~N}$ J Alp, ${ }^{3} \mathrm{U}$ Schotten, ${ }^{1} \mathrm{~B}$ Casadei. ${ }^{1}$ University of Oxford, John Radcliffe Hospital, Oxford, UK; ${ }^{2}$ First Department of Cardiology, University of Athens, Athens, Greece; ${ }^{3}$ Department of Physiology, University of Maastricht, Maastricht, The Netherlands

Background Atrial fibrillation (AF) is the most common sustained cardiac arrhythmia and is associated with altered nitric oxide (NO)redox balance. The molecular mechanisms and implications of this phenomenon in the management of patients with AF are poorly understood. Statins improve NO-redox imbalance and decrease the occurrence of postoperative AF but are less effective in the secondary prevention of AF, suggesting that the sources of reactive oxygen species might vary with the substrate and duration of AF.

Methods and Results We investigated atrial tissue from 130 patients undergoing cardiac surgery (26 with permanent AF, 32 who developed AF post-operatively and 72 who were in normal sinus rhythm before and after surgery), and from goats in sinus rhythm (SR, $\mathrm{n}=19$ ) with or without atrial structural remodelling secondary to surgical AV block (AVB, $\mathrm{n}=10)$ or after 2 weeks $(2 \mathrm{~W}, \mathrm{n}=15)$ or 6 months $(6 \mathrm{M}, \mathrm{n}=10)$ of pacing-induced AF. Atrial NADPH oxidase activity (chemiluminescence and 2-OH ethidium, Abstract 142 figures 1 and 2), NOX2 \& p22phox protein level were increased after $2 \mathrm{~W}-\mathrm{AF}$ and in patients who developed AF post-operatively $(n=32)$. In contrast, the increased superoxide production in atrial tissue from goats with AVB or 6M-AF was exclusively driven by mitochondrial oxidases and uncoupled NOS (secondary to a reduction in atrial $\mathrm{BH} 4$ level and an increase in arginase activity). These findings were recapitulated in the right atrial appendage of patients. Increase in basal superoxide production in postoperative AF was associated with an apocynin-reversible increase in NADPH oxidase activity and protein level of the NOX2 and p22phox subunits. NOS activity remained coupled despite the increase in superoxide production. In line with this, atrial $\mathrm{BH} 4$ content was unaltered. In contrast, in patients with permanent AF, increased superoxide production was not reversed by apocynin, and was maintained by mitochondrial oxidases and uncoupled NOS (secondary to BH4 deplition). Ex-vivo inhibition of HMG-CoA reductase with atorvastatin $(20 \mu \mathrm{Mol} / \mathrm{l})$ inhibited NADPH oxidase activity (via reducing activity of Rac1 and membrane translocation of cytosolic subunit p47phox and p67phox of NADPH oxidase) and caused a mevalonate-reversible reduction in 